Research Paper

\title{
Association between the KRAS Gene Polymorphisms and Papillary Thyroid Carcinoma in a Chinese Han Population
}

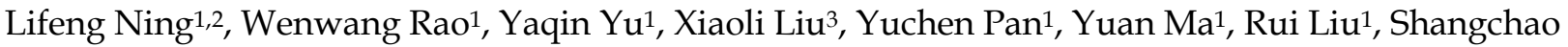 \\ Zhang ${ }^{1}$, Hui Sun ${ }^{3}$, Qiong Yu ${ }^{1 凶}$ \\ 1. Department of Epidemiology and Biostatistics, School of Public Health, Jilin University, Changchun, Jilin Province, China \\ 2. National Research Institute for Family Planning, Beijing, China. \\ 3. Jilin Provincial Key Laboratory of Surgical Translational Medicine, Department of Thyroid and Parathyroid Surgery, China-Japan Union Hospital, Jilin \\ University, Changchun, Jilin Province, China. \\ $\square$ Corresponding author: yuqiong@jlu.edu.cn; Tel.: +86 43185619451
}

(C) Ivyspring International Publisher. Reproduction is permitted for personal, noncommercial use, provided that the article is in whole, unmodified, and properly cited. See http://ivyspring.com/terms for terms and conditions.

Received: 2016.06.17; Accepted: 2016.10.10; Published: 2016.12.09

\begin{abstract}
Several studies have reported the association between MAPK signaling pathway gene polymorphisms and papillary thyroid carcinoma (PTC). KRAS gene, an oncogene from the mammalian RAS gene family plays an important role in the MAPK pathway. This study aimed to identify the potential association of KRAS gene polymorphisms with susceptibility to PTC in a Han Chinese population.

A total of 861 patients with PTC, 562 disease controls with nodular goiter and 897 healthy controls were recruited. Four tagSNP polymorphisms (rs12427141, rs712, rs7315339 and rs7960917) of KRAS gene were genotyped by matrix-assisted laser desorption/ionization time of flight mass spectrometry (MALDI-TOF-MS). Statistical analyses and haplotype estimations were conducted using Haploview and Unphased softwares.

Only significant differences were observed in genotypic frequencies of the rs 7315339 polymorphism $\left(\chi^{2}\right.$ $=7.234, \mathrm{df}=2, \mathrm{p}=0.027$ ) between PTC and disease controls. Statistically significant differences in both allelic and genotypic genotypes frequencies for rs712 (Genotype, $\chi^{2}=8.258, p=0.016$ ) and $r s 12427141$ (Allele, $\chi^{2}=3.992, p=0.046$; Genotype, $\chi^{2}=8.140, p=0.017$ ) were observed between PTC patients and controls. Haplotype analyses revealed higher frequencies of GA and TA haplotypes $(p=0.039$ and $\mathrm{p}=0.003$, respectively) from rs712- rs12427141 (two-SNP) or TGA and TTG haplotype containing the alleles from rs7960917, rs712 and rs 12427141, as well as the GAT haplotype containing the alleles from rs712, rs 12427141 and rs7315339 in PTC patients than in healthy controls $(p=0.042, p=0.037, p=0.027$, respectively). Inversely, the haplotype TTA from rs7960917, rs712 and rs12427141 or the haplotype TAC from rs712, rs 12427141 and rs7315339 was significantly less frequent in the PTC patients than in normal control $(p=0.003, p=0.003$, respectively).

These findings suggest the role of these KRAS gene variants in susceptibility to PTC. Moreover, significant differences of the KRAS gene polymorphisms may occur between nodular goiter and PTC.
\end{abstract}

Key words: KRAS; papillary thyroid carcinoma; polymorphisms; molecular epidemiology.

\section{Introduction}

Thyroid cancer (TC) is the most common endocrine malignancy that accounts for $1 \%$ of all cancers, about $95 \%$ of endocrine cancers and $60 \%$ of all death from endocrine, Epidemiological studies have reported a progressive increase in the overall incidence in most countries [1]. For example, the changes in the incidence of TC in the United States between 1975 and 2009 was from 4.9 to 14.3 per 100000 individuals. However, the mortality of TC has remained stable (approximately 0.5 deaths per 100 
000) [2]. In China, the TC incidence was increased 2.35 times from 1988 to 2009, with an average annual increase of $5.92 \%$. Moreover, the female TC incidence has been ranked among the top 10 cancer profile between 2005 and 2010 [3, 4]. However, the relative increase has been larger in men than women, showing that the increased incidence in men was almost 3 times greater than that of women[5] .

Interestingly, virtually all of the increased diagnosis of TC is attributable to an increase in papillary thyroid cancer (PTC), which accounts for more than $80 \%$ of all thyroid malignancies [6]. PTC is a well-differentiated thyroid cancer stemmed from follicular epithelial cells and characterized by distinctive nuclear features $[7,8]$. The cellular features of PTC are amphophilic, finely granular cytoplasm, large pale nuclei, nuclear grooves, and psammoma bodies. It has been known that PTC is a multifactorial disease affected by both genetic and environmental factors such as iodine intake or radiation exposure [9, 10].However, their relative contributions and the molecular mechanisms are still inconclusive [11].

Several studies have reported the association between MAPK signaling pathway gene polymorphisms and PTC $[12,13]$. KRAS gene, an oncogene from the mammalian RAS gene family that plays an important role in the MAPK pathway, encodes a protein that is a member of the small GTPase superfamily [14, 15]. The protein product of the normal KRAS gene performs an essential function in normal tissue signaling, and regulates a variety of biological processes, including cellular proliferation and apoptosis [16]. The mutation of KRAS gene is an essential step in the development of many cancers [17], particularly the mutation in the coding region of the KRAS gene, one of the major oncogenic mutation found in human cancer, which result in hyper-activation of the protein [18].

The expression of KRAS can be regulated on the gene level and affected by a single nucleotide polymorphism (SNP) located within the promoter or other regulatory regions [19]. The genotype and allele of SNP in the carcinogenesis-related genes might provide a simple and valuable method to predict the risk and the prognosis of cancer [20]. However, for a given set of markers spanning a gene, haplotype data usually provide more information than a single marker or even a group of markers analyzed separately. In the present study, we had chosen 4 tag polymorphism markers at the KRAS locus in our Chinese population and hypothesized that the KRAS gene variants may play a role in susceptibility to PTC. In addition, inflammation in thyroid gland such as nodular goiter [21, 22] has been confused with TC. Moreover, nodular goiter has shown the potential for malignancy and may be a risk factor for the development of TC [23-25] .Therefore, we had also recruited a group of nodular goiter as the disease controls and examined whether nodular goiter may be a transition stage for the occurrence of PTC.

\section{Methods}

\section{Subjects}

Eight hundred and sixty-one patients were recruited in the China-Japan Union Hospital of Jilin University from January 2011 to December 2013. Patients were diagnosed on the basis of pathological results of ultrasonography-guided, fine needle aspiration biopsy or resected specimens according to the revised American Thyroid Association management guidelines for patients with PTC. The exclusion criteria in the PTC group were patients with concomitant diagnosis of other cancers.

Eight hundred and ninth-seven healthy individuals were enrolled from a large-scale community-based cross-sectional survey of chronic disease and risk factors among adults in Jilin province during the same period. Any subjects with major medical illnesses were excluded, including metabolic syndrome, thyroid gland disease, or cancer.

To reveal the possible relationship between nodular goiter and PTC, five hundred and sixty-two patients with nodular goiter were recruited as the disease controls from the same hospital during the same period. Also, patients were diagnosed on the basis of pathological results according to the revised American Thyroid Association management guidelines.

All subjects were Han Chinese from Northern China and gender distributions in three groups were similar. They gave written informed consent to participate in the study, which was approved by the ethics committee of the school of Public Health, Jilin University.

\section{DNA extraction and genotyping analysis}

Genomic DNA was extracted from the EDTA-coated venous blood samples by using ClotBlood DNA Kit (Cwbio, Beijing) and then tested using an ultraviolet spectrophotometer (Beckman, USA) for DNA concentrations.

The KRAS gene contains 6 exons and is located at 12p12.1. The single nucleotide polymorphisms (SNPs) were the source of the HapMap database (http://www.hapmap.org). We selected four tag SNPs using Haploview version 4.2 [26] including rs12427141 (intron), rs712 (3'UTR), rs7315339 (intron), rs7960917 (3'UTR) (Population: CHB; R ${ }^{2}$ cutoff 0.8; MAF $\left.>0.1 ; D^{\prime}=1\right)$. The primers of four Tag SNPs were designed by Assay Designer3.1 (Table 1). SNP 
genotyping was conducted by using an iPLEX Gold reagent set (Sequenom, San Diego, CA, USA) containing a PCR enzyme GPR, PCR accessory set, SpectroCHIP II resin kit, and iPLEX Gold reagent kit. The standard polymerase chain reaction (PCR) was performed and PCR products were assayed by the matrix-assisted laser desorption/ionization time of flight mass spectrometry (MALDI-TOF-MS).

\section{Statistical analysis}

Deviations from Hardy-Weinberg disequilibrium(HWD) was calculated by Chi-square $\left(\chi^{2}\right)$ goodness-of-fit test. Group differences were compared using Student's two-sample t-test or one-way analysis of variance (ANOVA) for continuous variables and Chi-squared for categorical variables. Differences of the $4 K R A S$ gene allele and genotype frequencies between groups were evaluated using Chi-square test. Codominant, dominant, recessive, and overdominant genetic models of inheritance were chosen to evaluate the associations of each SNP using the SNPStats program (http://bioinfo.iconcologia.net/SNPStats). The best model of inheritance for each SNP was select based on the smallest value of akaike information criterion (AIC) and bayesian information criterion (BIC). Between four KRAS markers, pairwise linkage disequilibrium (LD) was analyzed in three groups. Haplotype block, haplotype frequency, and haplotype association were calculated via unphase 3.1 [27]. We conducted a $2 \sim 3$-window fashion analysis. Less than $1 \%$ rare haplotypes were ruled out from the association analysis. 10,000 permutations were executed to adjust for multiple testing.

In this study, we defined the statistical power of the sample as the probability that the test correctly detects true differences of the sample. We then calculated the power using Quanto 1.2.4 Software [28], with a PTC population prevalence of 3.7 per 100 000 [5] and known risk allele frequencies. All data analyses were performed using SPSS18.0 software unless otherwise specified. Data are presented as mean $\pm S D$. Two-tailed significance values were used and significance levels were set at 0.05 .

Table 1. Primer sequences for the KRAS gene analysis.

\begin{tabular}{|c|c|c|}
\hline SNPS & & Primer sequence \\
\hline \multirow[t]{2}{*}{ rs12427141 } & Forward & 5'-ACGTTGGATGCAAGACAGAGTTTAATACGGG-3 \\
\hline & Reverse & 5'-ACGTTGGATGGGGACATAGTAACTACCCAG-3' \\
\hline \multirow[t]{2}{*}{ rs7960917 } & Forward & 5'-ACGTTGGATGTAGTCCCTCCCCATTTTGAC-3' \\
\hline & Reverse & 5'-ACGTTGGATGGAGAGTTTCACAGCATGGAC-3' \\
\hline \multirow[t]{2}{*}{ rs712 } & Forward & 5'-ACGTTGGATGCCAAAACTCTGGGAATACTG-3' \\
\hline & Reverse & 5'-ACGTTGGATGGCAGACTGTTAGCTTTTACC-3' \\
\hline \multirow[t]{2}{*}{ rs7315339 } & Forward & 5'-ACGTTGGATGGAAAAGCTAGTAAGGAGTGG-3' \\
\hline & Reverse & 5'-ACGTTGGATGGATGGTAGATGGTAATTCAG-3' \\
\hline
\end{tabular}

\section{Results}

\section{Subject characteristics}

Demographic characteristics of three groups showed that no differences were observed in gender among three groups and in age between PTC patients and healthy controls (all, $P>0.05$ ). However, the mean age of nodular goiter patients (mean age $=48.51 \pm 10.103$ ) were significantly older than both PTC patients (mean age $=43.76 \pm 9.302$ ) and healthy controls (mean age $=43.73 \pm 9.061$ ). Further, we found no differences between genotypes on any of the demographic parameters (all, $P>0.05$ ).

\section{The Hardy-Weinberg Equilibrium Analysis}

The genotype distributions and allele frequencies of 4 SNPs located in the KRAS gene showed that in the three groups the deviation was not detected in HWE (all p > 0.05) except for rs12427141 and rs712 in normal control group $\left(\chi^{2}=6.141\right.$, $\left.P=0.013 ; \chi^{2}=14.786, P<0.001\right)$, rs7315339 in PTC group $\left(\chi^{2}=7.232, P=0.007\right)$, and rs7960917 in the three groups (all $P<0.05$ ). Thus, we chose $10 \%$ samples from normal control group randomly to review genotyping results, showing that the accuracy rate were more than $99 \%$.

\section{The Allele and Genotype Analysis}

Between PTC patients and controls, significant differences in the genotype distributions and allele frequencies were observed for rs12427141 (genotype $\chi^{2}=8.140, \quad P=\mathbf{0 . 0 1 7} ; \quad$ allele $\chi^{2}=3.992, \quad P=\mathbf{0 . 0 4 6}$ ). Between the other three SNPs and PTC patients, the allelic or genotypic association was not found (all $\mathrm{p}>0.05)$ except for the genotype distribution of rs712 $\left(\chi^{2}=8.258, \quad P=\mathbf{0 . 0 1 6}\right) \quad$ (Table 2). Inheritance modeling based on the smallest value of AIC and BIC suggested a recessive model for rs12427141, an overdominant model for rs712, and a recessive model for rs7315339 by comparing PTC and control groups. Tests for association with individual SNPs showed significance at 2 SNPs (rs12427141， $P=\mathbf{0 . 0 0 6 2} ; \quad$ rs7315339, $P=\mathbf{0 . 0 2 8}$ ) under the recessive model and 1 SNP (rs712, $P=\mathbf{0 . 0 0 4 5}$ ) under the overdominant model.

Table 3 shows that only the genotype distribution of rs7315339 was found to be significant between PTC and nodular goiter groups $\left(\chi^{2}=7.234, P=0.027\right)$. Between PTC and nodular goiter groups, there were no allelic or genotypic differences in the other SNPs (all p>0.05) (Table 3). Inheritance modeling based on the smallest value of AIC and BIC suggested a recessive model for 
rs12427141, a recessive model for rs712, and an overdominant model for rs7315339 by comparing nodular goiter and control groups. Interestingly, one KRAS SNP, rs7315339, showed a statistically significant association with the nodular goiter risk under the overdominant model $(P=\mathbf{0 . 0 4 6})$.

In addition, there was no allelic or genotypic differences in all four SNPs between patients with nodular goiter and healthy controls (all p>0.05) (Table 4).

\section{The Haplotype Analysis}

Since a polygenic disease may be associated with a specific combination of alleles, rather than a single allele, we constructed haplotypes [29]. There was a significant overall difference between PTC and healthy controls (all, $\mathrm{p}<0.05$ ). Two-four SNP sliding window haplotype analysis indicated that among
two-SNP haplotypes, the GA haplotype frequency was greater, but TA less in PTC patients than in healthy controls $\left(\chi^{2}=4.250, P=0.039 ; \chi^{2}=8.706, P=0.003\right.$, respectively). Among 3-SNP haplotypes, the haplotypes TGA and TTG containing the alleles from rs7960917, rs712 and rs12427141, as well as the haplotype GAT containing the alleles from rs712, rs12427141 and rs7315339 were significantly more common in patients with PTC than in healthy control subjects $\left(\chi^{2}=4.150, P=0.042\right.$ for TGA; $\chi^{2}=4.359, P=0.037$ for TTG; $\chi^{2}=4.908, P=0.027$ for GAT, respectively).

On the contrary, the haplotype TTA from rs7960917, rs712 and rs12427141 or the haplotype TAC from rs712, rs12427141 and rs7315339 was significantly less frequent in the PTC patients than in normal control $\left(\chi^{2}=8.528, P=0.003\right.$ for TTA; $\chi^{2}=9.105$, $P=0.003$ for TAC) (Table 5).

Table 2. The genotype and allele frequencies of SNPs in KRAS gene between patients with PTC and normal control.

\begin{tabular}{|c|c|c|c|c|c|c|c|c|c|c|}
\hline \multirow[b]{2}{*}{ TagSNP * } & \multirow[b]{2}{*}{ Group $(\mathbf{n}) \dagger$} & \multicolumn{5}{|c|}{ Genotypic association (\%) } & \multicolumn{4}{|c|}{ Allelic association (\%) } \\
\hline & & $1 / 1$ & $1 / 2$ & $2 / 2$ & $\mathrm{X} 2$ & $\mathbf{P}$ & 1 & 2 & $\mathrm{X} 2$ & $\mathbf{P}$ \\
\hline \multirow[t]{2}{*}{ rs7960917 } & PTC (861) & $701(81.4)$ & $160(18.6)$ & $0(0.0)$ & 0.754 & 0.385 & $1562(90.7)$ & $160(9.3)$ & 0.673 & 0.412 \\
\hline & NC (890) & $710(79.8)$ & $180(20.2)$ & $0(0.0)$ & & & $1600(89.9)$ & $180(10.1)$ & & \\
\hline \multirow[t]{2}{*}{ rs712 } & PTC (858) & $527(61.4)$ & $289(33.7)$ & $42(4.9)$ & 8.258 & 0.016 & $1343(78.3)$ & $373(21.7)$ & 2.470 & 0.116 \\
\hline & NC (886) & $591(66.7)$ & $243(27.4)$ & $52(5.9)$ & & & $1425(80.4)$ & $347(19.6)$ & & \\
\hline \multirow[t]{2}{*}{ rs12427141 } & PTC (860) & $632(73.5)$ & $211(24.5)$ & $17(2.0)$ & 8.140 & 0.017 & $1475(85.8)$ & $245(14.2)$ & 3.992 & 0.046 \\
\hline & NC (894) & $685(76.6)$ & $204(22.8)$ & $5(0.6)$ & & & $1574(88.0)$ & $214(12.0)$ & & \\
\hline \multirow[t]{2}{*}{ rs7315339 } & PTC (824) & $665(80.7)$ & $158(19.2)$ & $1(1.0)$ & 5.795 & 0.055 & $1488(90.3)$ & $160(9.7)$ & 2.960 & 0.085 \\
\hline & NC (858) & 667 (77.7) & $184(21.4)$ & $7(0.8)$ & & & $1518(88.5)$ & 198 (11.5) & & \\
\hline
\end{tabular}

* The allele of rs7960917 is T and C, the allele of rs712 is G and T, the allele of rs12427141 is G and A, the allele of rs7315339 is T and C.

†PTC means papillary thyroid carcinoma, NC means normal control.

Table 3. The genotype and allele frequencies of SNPs in KRAS gene between patients with PTC and disease control.

\begin{tabular}{|c|c|c|c|c|c|c|c|c|c|c|}
\hline \multirow[b]{2}{*}{ TagSNP * } & \multirow[b]{2}{*}{ Group (n) $\dagger$} & \multicolumn{5}{|c|}{ Genotypic association (\%) } & \multicolumn{4}{|c|}{ Allelic association (\%) } \\
\hline & & $1 / 1$ & $1 / 2$ & $2 / 2$ & $\mathrm{X}^{2}$ & $P$ & 1 & 2 & $\mathrm{X}^{2}$ & $P$ \\
\hline \multirow[t]{2}{*}{ rs7960917 } & PTC (861) & $701(81.4)$ & $160(18.6)$ & $0(0.0)$ & 0.476 & 0.490 & $1562(90.7)$ & $160(9.3)$ & 0.429 & 0.512 \\
\hline & DC (560) & $464(82.9)$ & $96(17.1)$ & $0(0.0)$ & & & $1024(91.4)$ & $96(8.6)$ & & \\
\hline \multirow[t]{2}{*}{ rs712 } & PTC (858) & $527(61.4)$ & $289(33.7)$ & $42(4.9)$ & 3.977 & 0.137 & $1343(78.3)$ & $373(21.7)$ & 2.547 & 0.110 \\
\hline & DC (551) & $366(66.4)$ & $158(28.7)$ & $27(4.9)$ & & & $890(80.8)$ & $212(19.2)$ & & \\
\hline \multirow[t]{2}{*}{ rs12427141 } & PTC (860) & $632(73.5)$ & $211(24.5)$ & $17(2.0)$ & 1.628 & 0.443 & $1475(85.8)$ & $245(14.2)$ & 1.582 & 0.209 \\
\hline & DC (560) & $427(76.3)$ & $125(22.3)$ & $8(1.4)$ & & & $979(87.4)$ & $141(12.6)$ & & \\
\hline \multirow[t]{2}{*}{ rs7315339 } & PTC (824) & $665(80.7)$ & $158(19.2)$ & $1(1.0)$ & 7.234 & 0.027 & $1488(90.3)$ & $160(9.7)$ & 0.001 & 0.979 \\
\hline & DC (527) & $431(81.8)$ & 90 (17.1) & $6(1.1)$ & & & $952(90.3)$ & $102(9.7)$ & & \\
\hline
\end{tabular}

* The allele of rs7960917 is T and C, the allele of rs712 is G and T, the allele of rs12427141 is G and A, the allele of rs7315339 is T and C.

†PTC means papillary thyroid carcinoma, DC means disease control.

Table 4. The genotype and allele frequencies of SNPs in KRAS gene between patients with nodular goiter and normal control.

\begin{tabular}{|c|c|c|c|c|c|c|c|c|c|c|}
\hline \multirow[b]{2}{*}{ TagSNP * } & \multirow[b]{2}{*}{ Group (n) ${ }^{\dagger}$} & \multicolumn{5}{|c|}{ Genotypic association (\%) } & \multicolumn{4}{|c|}{ Allelic association (\%) } \\
\hline & & $1 / 1$ & $1 / 2$ & $2 / 2$ & $\mathrm{X}^{2}$ & $P$ & 1 & 2 & $\mathrm{X}^{2}$ & $P$ \\
\hline \multirow[t]{2}{*}{ rs7960917 } & DC (560) & $464(82.9)$ & $96(17.1)$ & $0(0.0)$ & 2.118 & 0.146 & $1024(91.4)$ & $96(8.6)$ & 1.896 & 0.169 \\
\hline & NC (890) & $710(79.8)$ & $180(20.2)$ & $0(0.0)$ & & & $1600(89.9)$ & $180(10.1)$ & & \\
\hline \multirow[t]{2}{*}{ rs712 } & DC (551) & $336(66.4)$ & $158(28.7)$ & $27(4.9)$ & 0.774 & 0.679 & $890(80.8)$ & $212(19.2)$ & 0.052 & 0.820 \\
\hline & NC (886) & $591(66.7)$ & $243(27.4)$ & $52(5.9)$ & & & $1425(80.4)$ & 347 (19.6) & & \\
\hline \multirow[t]{2}{*}{ rs12427141 } & DC (560) & $427(76.3)$ & $125(22.3)$ & $8(1.4)$ & 2.954 & 0.228 & $979(87.4)$ & $141(12.6)$ & 0.247 & 0.619 \\
\hline & NC (894) & $685(76.6)$ & $204(22.8)$ & $5(0.6)$ & & & $1574(88.0)$ & $214(12.0)$ & & \\
\hline \multirow[t]{2}{*}{ rs7315339 } & DC (527) & $431(81.8)$ & $90(17.1)$ & $6(1.1)$ & 4.184 & 0.123 & $952(90.3)$ & $102(9.7)$ & 2.342 & 0.126 \\
\hline & NC (858) & 667 (77.7) & $184(21.4)$ & $7(0.8)$ & & & $1518(88.5)$ & $198(11.5)$ & & \\
\hline
\end{tabular}

* The allele of rs7960917 is T and C, the allele of rs712 is G and T, the allele of rs12427141 is G and A, the allele of rs7315339 is T and C.

†DC means disease control, NC means normal control. 
Table 5. The haplotypes association analysis of KRAS gene between patients with PTC and normal control.

\begin{tabular}{|c|c|c|c|c|}
\hline \multirow{2}{*}{ Haplotypes ${ }^{*}$} & \multicolumn{3}{|c|}{ Haplotypes Frequency 2} & \multirow[t]{2}{*}{$P$} \\
\hline & PTC & NC & & \\
\hline rs712- rs12427141 & & & Global $\chi 2=13.920$ & 0.003 \\
\hline GG & 0.779 & 0.769 & 0.670 & 0.413 \\
\hline GA & 0.023 & 0.013 & 4.250 & 0.039 \\
\hline TG & 0.101 & 0.088 & 1.274 & 0.259 \\
\hline TA & 0.097 & 0.130 & 8.706 & 0.003 \\
\hline $\begin{array}{l}\text { rs7960917 } \\
\text { - rs712-rs12427141 }\end{array}$ & & & Global $\chi 2=19.517$ & 0.003 \\
\hline TGG & 0.766 & 0.76 & 0.233 & 0.629 \\
\hline TGA & 0.022 & 0.012 & 4.150 & 0.042 \\
\hline TTG & 0.011 & 0.005 & 4.359 & 0.037 \\
\hline TTA & 0.099 & 0.130 & 8.528 & 0.003 \\
\hline CGG & 0.015 & 0.009 & 2.234 & 0.135 \\
\hline CGA & 2.23E-18 & $4.36 \mathrm{E}-04$ & 0.445 & 0.505 \\
\hline CTG & 0.088 & 0.083 & 0.213 & 0.645 \\
\hline $\begin{array}{l}\text { rs712-rs12427141 } \\
\text {-rs7315339 }\end{array}$ & & & Global $\chi 2=15.570$ & 0.016 \\
\hline GGT & 0.748 & 0.743 & 0.144 & 0.705 \\
\hline GGC & 0.032 & 0.026 & 1.107 & 0.293 \\
\hline GAT & 0.023 & 0.012 & 4.908 & 0.027 \\
\hline GAC & 0 & $1.50 \mathrm{E}-18$ & $-9.01 \mathrm{E}-08$ & 1 \\
\hline TGT & 0.014 & 0.013 & 0.103 & 0.749 \\
\hline TGC & 0.086 & 0.075 & 1.278 & 0.258 \\
\hline TAC & 0.097 & 0.130 & 9.105 & 0.003 \\
\hline
\end{tabular}

"Haplotypes with frequency $>1 \%$ in all subjects were lists.

\section{The power Analysis}

This total sample had $0.93-0.99$ power for rs7960917, 0.98-0.99 for rs712, and 0.97-0.99 for rs12427141, and 0.93-0.99 for rs7315339 to detect dominant polymorphic inheritance in the PTC patients and normal controls, with an odds ratio (OR) of 1.5-2.0 ( $\mathrm{a}=0.05$, two-tailed test). Similarly, this total sample had 0.84-0.99 power for rs7960917, 0.95-0.99 for rs712, and 0.92-0.99 for rs12427141, and 0.87-0.99 for rs7315339 to detect dominant polymorphic inheritance in the nodular goiter patients and normal controls, with an odds ratio (OR) of 1.5-2.0 ( $\mathrm{a}=0.05$, two-tailed test). In addition, this total sample had 0.85-0.99 power for rs7960917, 0.95-0.99 for rs712, and 0.94-0.99 for rs12427141, and 0.86-0.99 for rs7315339 in the PTC and nodular goiter patients.

\section{Discussion}

To our knowledge, this is the first study to investigate the relationships between KRAS gene haplotypes and PTC or nodular goiter patients in a Chinese population. This study had three major findings. (1) The KRAS rs12427141, or probably rs712 may contribute to the susceptibility to PTC. (2) There was a significant difference in the genotype distribution of KRAS rs7315339 between PTC and nodular goiter groups. (3) An association between KRAS gene haplotypes and PTC was found.

It is known that the oncogene mutations of the RAS family gene are associated with the development of thyroid cancer [30-32]. KRAS gene variations are the predominated RAS mutations in most types of cancer [24]. KRAS point mutations have been found in $20 \%-40 \%$ of PTC patients across different studies [33]. Converging lines of evidence have suggested that KRAS genes were activated at a relatively high frequency in human thyroid cancers. The most mutations of KRAS gene were located primarily in exon2, exon3 and exon4 [17, 34-38]. However, some studies did not find point mutations in these regions of KRAS gene [39-41].

Numerous RAS gene mutations have been found to have an essential role in the initiation of tumorigenesis and have been identified in diverse thyroid cancers [42-44], particularly in PTC [45]. In thyroid cancer, RAS gene is a unanimous dual activator of the MAPK and PI3K-AKT pathways. RAS gene mutations may promote thyroid tumorigenesis through the verified Ras-Raf-MEK-MAP/ERK pathway (also named MAP kinase pathway) or through its interaction with the PI3K-AKT pathway [46]. After these two pathways are activated by the RAS gene mutations, they may promote the development and progression of thyroid cancer, which was supported by the experiments of transgenic mice with the deletion of Pten and knock-in of KRASG12D [47].

Several studies revealed that the rs712 polymorphism in KRAS gene was associated with cancer risk [48-50]. The most recent meta-analysis also showed that KRAS gene were strongly associated with cancer in Chinese population [51]. These studies have our results to some extent. In addition, Jin et al. have reported recently that the KRAS rs712 polymorphism was not associated with PTC risk in a Chinese population [52], which is disagreement with our current study. One possible explanation for the inconsistency is a difference in geographical regions and sample size between our current study and Jin et al.

Several limitations need to be interpreted with caution. First, the TagSNP selection method in our study may ignore another functional SNP. However, this method was used in a large number of literatures and also was in accordance with statistical principles. Second, despite the presence of departures from HWE, our study has eliminated genotyping errors, the chance or failure of the requisite assumptions of HWE. Third, many subjects were not included in the genotype analysis of 4 SNPs, and this might bring about the bias in the statistical analysis due to the imbalance in the number of 4 SNPs in our current study.

In conclusion, our findings implicate that $K R A S$ genotype has an effective function on increased risk 
for PTC based on individual genotype and haplotype analyses. We observed a potential genetic association of KRAS with the risk for PTC in genetically homogeneous Han Chinese population, especially the KRAS gene polymorphism rs12427141, or probably rs712. Furthermore, there was a significant difference in the genotype distribution of KRAS rs7315339 between PTC and nodular goiter groups, suggesting the difference in genetic makeup of the two diseases. Nevertheless, our present study remain a preliminary stage, due to the limited sample size, the low statistical power, and the poor coverage of genetic variations in $K R A S$, which recommend to further validate the accuracy and specificity of the association in larger samples of PTC patients and/or in different ethnic populations.

\section{Abbreviations}

PTC: papillary thyroid carcinoma, KRAS: Kirsten rat sarcoma viral oncogene homolog; SNP: Single nucleotide polymorphism; MALDI-TOF/TOF-MS: Matrix-assisted laser desorption ionization time-of-flight tandem mass spectrometry; UTR: Untranslated regions; PCR: Polymerase Chain Reaction; HWD: Hardy W'einberg disquilibrium; AIC: akaike information criterion; BIC: bayesian information criterion; LD: Linkage disequilibrium; GWAS: Genome wide association study.

\section{Acknowledgments}

This work was supported by Norman Bethune Program of Jilin University [2015227], the Young Scholars Program of Norman Bethune Health Science Center of Jilin University (No.2013202018) and the young Program of Jilin Provincial Health Department (2014Q027).

\section{Competing interests}

The authors have declared that no competing interest exists.

\section{References}

1. Albores-Saavedra J, Henson DE, Glazer E, Schwartz AM. Changing patterns in the incidence and survival of thyroid cancer with follicular phenotype-papillary, follicular, and anaplastic: a morphological and epidemiological study. Endocr Pathol. 2007; 18: 1-7.

2. Davies L, Welch HG. Current thyroid cancer trends in the United States. JAMA Otolaryngol Head Neck Surg. 2014; 140: 317-22.

3. Sun J, Xu X, Cai Q. Epidemiological Study on Thyroid Cancer in China. China Cancer. 2013; 9: 690-3.

4. Chen W, Zheng R, Zhang S. Cancer Incidence, Mortality and Trend in China. journal1. 2014; 32: 65-71.

5. WHO: Geneva, Switzerland. Globocan2012: Estimated cancer incidence, mortality and prevalence worldwide in 2012. Revised 20 August 2015. http://globocaniarcfr/Pages/fact_sheets_populationaspx.

6. WHO: Geneva, Switzerland. World cancer report 2014. Revised 20 August 2015. http://www.iarc.fr/en/publications/books/wcr/wcr-order.php.

7. Gu Y, Yu Y, Ai L, Shi J, Liu X, Sun H, et al. Association of the ATM gene polymorphisms with papillary thyroid cancer. Endocrine. 2014; 45: 454-61.
8. Ai L, Yu Y, Liu X, Wang C, Shi J, Sun H, et al. Are the SNPs of NKX2-1 associated with papillary thyroid carcinoma in the Han population of Northern China? Front Med. 2014; 8: 113-7.

9. Nix P, Nicolaides A, Coatesworth A. Thyroid cancer review 1: presentation and investigation of thyroid cancer. Int J Clin Pract. 2005; 59: 1340-4.

10. Meinhold CL, Ron E, Schonfeld SJ, Alexander BH, Freedman DM, Linet MS, et al. Nonradiation risk factors for thyroid cancer in the US Radiologic Technologists Study. Am J Epidemiol. 2010; 171: 242-52.

11. Santoro M, Vecchio G. Thyroid cancer: A molecular perspective. Mol Cell Endocrinol. 2010; 321:1-2.

12. Nikiforov YE. Thyroid carcinoma: molecular pathways and therapeutic targets. Modern Pathol. 2008; 21: S37-S43.

13. Knauf JA, Fagin JA. Role of MAPK pathway oncoproteins in thyroid cancer pathogenesis and as drug targets. Curr Opin Cell Biol. 2009; 21: 296-303.

14. Xing M. BRAF mutation in thyroid cancer. Endocr Relat Cancer. 2005; 12: 245-62.

15. Saavedra HI, Knauf JA, Shirokawa JM, Wang J, Ouyang B, Elisei R, et al. The RAS oncogene induces genomic instability in thyroid PCCL3 cells via the MAPK pathway. Oncogene. 2000; 19: 3948-54.

16. Karnoub AE, Weinberg RA. Ras oncogenes: split personalities. Nat Rev Mol Cell Bio. 2008; 9: 517-31.

17. Kranenburg $\mathrm{O}$. The KRAS oncogene: past, present, and future. BBA-Rev Cancer. 2005; 1756: 81-2.

18. Kim M, Chen X, Chin L, Paranjape T, Speed W, Kidd K, et al. Extensive sequence variation in the $3^{\prime}$ untranslated region of the KRAS gene in lung and ovarian cancer cases. Cell Cycle. 2014; 13: 1030-40.

19. Chin LJ, Ratner E, Leng S, Zhai R, Nallur S, Babar I, et al. A SNP in a let-7 microRNA complementary site in the KRAS 3' untranslated region increases non-small cell lung cancer risk. Cancer Res. 2008; 68: 8535-40.

20. Kwon KH, Lee YC, Chung JH, Eun YG. Association study of chemokine (C-C motif) ligand 5 gene polymorphism and papillary thyroid cancer. J Invest Surg. 2013; 26: 319-24.

21. Mantovani A, Allavena P, Sica A, Balkwill F. Cancer-related inflammation. Nature. 2008; 454: 436-44

22. Guarino V, Castellone MD, Avilla E, Melillo RM. Thyroid cancer and inflammation. Mol Cell Endocrinol. 2010; 321: 94-102.

23. Thavarajah S, Weber F. Genetic Background May Confer Susceptibility to PTC in Benign Multinodular Thyroid Disease. J Cancer Ther. 2012; 3: 997.

24. Forbes SA, Beare D, Gunasekaran P, Leung K, Bindal N, Boutselakis H, et al. COSMIC: exploring the world's knowledge of somatic mutations in human cancer. Nucleic Acids Res. 2015; 43: 805-11.

25. Arora N, Scognamiglio T, Zhu B, Fahey TJ, 3rd. Do benign thyroid nodules have malignant potential? An evidence-based review. World J Surg. 2008; 32: 1237-46.

26. Barrett JC, Fry B, Maller J, Daly MJ. Haploview: analysis and visualization of LD and haplotype maps. Bioinformatics. 2005; 21: 263-5.

27. Dudbridge F. Likelihood-based association analysis for nuclear families and unrelated subjects with missing genotype data. Hum Hered. 2008; 66: 87-98.

28. Gauderman WJ. Sample size requirements for association studies of gene-gene interaction. Am J Epidemiol. 2002; 155: 478-84.

29. Gabriel SB, Schaffner SF, Nguyen H, Moore JM, Roy J, Blumenstiel B, et al. The structure of haplotype blocks in the human genome. Science. 2002; 296: 2225-9.

30. Tóbiás B, Balla B, Kósa P J, Horányi J, Takács I, Bölöny E, et al. Comprehensive examination of somatic oncogene mutation in normal and pathologic thyroid tissues. Orvosi Hetilap. 2011; 152: 672-7.

31. Park JY, Kim WY, Hwang TS, Lee SS, Kim H, Han HS, et al. BRAF and RAS mutations in follicular variants of papillary thyroid carcinoma. Endocr Pathol. 2013; 24: 69-76.

32. Khan MS, Pandith AA, ul Hussain M, Iqbal M, Khan NP, Wani KA, et al. Lack of mutational events of RAS genes in sporadic thyroid cancer but high risk associated with HRAS T81C single nucleotide polymorphism (case-control study). Tumor Biol. 2013; 34: 521-9.

33. Xing M. Molecular pathogenesis and mechanisms of thyroid cancer. Nat Rev Cancer. 2013; 13: 184-99.

34. Zou M, Baitei EY, Alzahrani AS, BinHumaid FS, Alkhafaji D, Al-Rijjal RA, et al. Concomitant RAS, RET/PTC, or BRAF mutations in advanced stage of papillary thyroid carcinoma. Thyroid. 2014; 24: 1256-66.

35. Schulten H-J, Salama S, Al-Ahmadi A, Al-Mansouri Z, Mirza Z, Al-Ghamdi K, et al. Comprehensive survey of HRAS, KRAS, and NRAS mutations in proliferative thyroid lesions from an ethnically diverse population. Anticancer Res. 2013; 33: 4779-84.

36. Park SJ, Sun JY, Hong K, Kwak JY, Kim EK, Chung WY, et al. Application of BRAF, NRAS, KRAS mutations as markers for the detection of papillary thyroid cancer from FNAB specimens by pyrosequencing analysis. Clin Chem Lab Med. 2013; 51: 1673-80.

37. Goutas N, Vlachodimitropoulos D, Bouka M, Lazaris AC, Nasioulas G, Gazouli M. BRAF and K-RAS mutation in a Greek papillary and medullary thyroid carcinoma cohort. Anticancer Res. 2008; 28: 305-8

38. Giordano TJ, Kuick R, Thomas DG, Misek DE, Vinco M, Sanders D, et al. Molecular classification of papillary thyroid carcinoma: distinct BRAF, RAS, and RET/PTC mutation-specific gene expression profiles discovered by DNA microarray analysis. Oncogene. 2005; 24: 6646-56.

39. Sadow PM, Heinrich MC, Corless CL, Fletcher JA, Nose V. Absence of BRAF, NRAS, KRAS, HRAS mutations, and RET/PTC gene rearrangements 
distinguishes dominant nodules in Hashimoto thyroiditis from papillary thyroid carcinomas. Endocr Pathol. 2010; 21: 73-9.

40. Matsuse M, Sasaki K, Nishihara E, Minami S, Hayashida C, Kondo H, et al. Copy number alteration and uniparental disomy analysis categorizes Japanese papillary thyroid carcinomas into distinct groups. PLos One.2012; 7:e36063.

41. Hou P, Xing M. Absence of Germline Mutations in Genes within the MAP Kinase Pathway in Familial Nonmedullary Thyroid Cancer. Cell Cycle. 2014; 5: 2036-9.

42. Zhu Z, Gandhi M, Nikiforova MN, Fischer AH, Nikiforov YE. Molecular profile and clinical-pathologic features of the follicular variant of papillary thyroid carcinoma An unusually high prevalence of ras mutations. Am J Clin Pathol. 2003; 120: 71-7.

43. Vasko V, Ferrand M, Di Cristofaro J, Carayon P, Henry J, De Micco C. Specific pattern of RAS oncogene mutations in follicular thyroid tumors. J Clin Endocr Metab. 2003; 88: 2745-52.

44. Rivera M, Ricarte-Filho J, Knauf J, Shaha A, Tuttle M, Fagin JA, et al. Molecular genotyping of papillary thyroid carcinoma follicular variant according to its histological subtypes (encapsulated vs infiltrative) reveals distinct BRAF and RAS mutation patterns. Modern pathol. 2010; 23: 1191-200.

45. Xing M. Recent advances in molecular biology of thyroid cancer and their clinical implications. Otolaryng Clin N Am. 2008; 41: 1135-46.

46. Hou P, Liu D, Shan $\mathrm{Y}, \mathrm{Hu}$ S, Studeman K, Condouris S, et al. Genetic alterations and their relationship in the phosphatidylinositol 3-kinase/Akt pathway in thyroid cancer. Clin Cancer Res. 2007; 13: 1161-70.

47. Miller KA, Yeager N, Baker K, Liao X-H, Refetoff S, Di Cristofano A. Oncogenic Kras requires simultaneous PI3K signaling to induce ERK activation and transform thyroid epithelial cells in vivo. Cancer Res. 2009; 69: 3689-94.

48. Yan L, Wang Q, Tian K. Association between the single nucleotide polymorphism of let-7 target gene KRAS-binding site rs712 and risk of glioma. Chin J Cancer Prev Treat. 2013; 20: 811-4.

49. Pan X, Sun R, Li Z, Guo X, Zhang Z, Qin H, et al. A let-7 KRAS rs712 polymorphism increases colorectal cancer risk. Tumor Biol. 2014; 35: 831-5.

50. Li Z, Pan X, Han B, Guo X, Zhang Z, Jia J, et al. A let-7 binding site polymorphism rs712 in the KRAS 3' UTR is associated with an increased risk of gastric cancer. Tumor Biol. 2013; 34: 3159-63.

51. Zhao $\mathrm{W}, \mathrm{Qu} X$, Xing $\mathrm{Z}$, Zhao $\mathrm{L}$, Qin L, Lv C. Association of rs712 polymorphism in Kras gene 3 -untranslated region and cancer risk: A meta-analysis. J BUON. 2015; 20: 309 .

52. Jin $\mathrm{H}$, Liang $\mathrm{Y}$, Wang $\mathrm{X}$, Zhu J, Sun R, Chen $\mathrm{P}$, et al. Association between a functional polymorphism rs712 within let-7-binding site and risk of papillary thyroid cancer. Med Oncol. 2014; 31: 1-5. 